\title{
Effect of ultrasonication on the size distribution and stability of cellulose nanocrystals in suspension: an asymmetrical flow field-flow fractionation study
}

\author{
Christoph Metzger $(\mathbb{D} \cdot$ Roland Drexel $\cdot$ Florian Meier $(\mathbb{D}) \cdot$ Heiko Briesen $(\mathbb{D}$
}

Received: 2 March 2021/Accepted: 26 August 2021/Published online: 8 September 2021

(C) The Author(s) 2021

\begin{abstract}
Cellulose nanocrystals (CNCs) are biobased building blocks for sustainable advanced materials with prospective applications in polymer composites, emulsions, electronics, sensors, and biomedical devices. However, their high surface area-to-volume ratio promotes agglomeration, which restrains their performance in size-driven applications, thereby hindering commercial CNC utilization. In this regard, ultrasonication is commonly applied to disperse CNCs in colloidal suspensions; however, ultrasonication methodology is not yet standardized and knowledge of the effects of ultrasound treatments on CNC size distribution is scarce. The major goals of this study were attributed to targeted breakage of CNC agglomerates and clusters by ultrasound. The evolution of particle size distribution and potential desulfation by ultrasonication as well as the long-term stability of ultrasonicated CNC suspensions were
\end{abstract}

Supplementary Information The online version contains supplementary material available at https://doi.org/10.1007/ s10570-021-04172-3.

C. Metzger $(\bowtie) \cdot$ H. Briesen

TUM School of Life Sciences Weihenstephan, Chair of

Process Systems Engineering, Technical University of

Munich, Freising, Germany

e-mail: christoph.metzger@tum.de

URL: http://svt.wzw.tum.de/

R. Drexel · F. Meier

Postnova Analytics GmbH, Rankinestr. 1,

86899 Landsberg am Lech, Germany investigated. Colloidal suspensions of sulfated CNCs were isolated from cotton $\alpha$-cellulose. Effects of ultrasonication on particle size distribution were determined by asymmetrical flow field-flow fractionation (AF4) coupled with on-line multi-angle light scattering and ultraviolet spectroscopy. These results were complemented with off-line dynamic light scattering. High ultrasound energy densities facilitated cumulative dispersion of CNC clusters. Consequently, the mean rod length decreased logarithmically from $178.1 \mathrm{~nm}$ at an ultrasound energy input of $2 \mathrm{~kJ} \mathrm{~g}^{-1}$ $\mathrm{CNC}$ to $141.7 \mathrm{~nm}(-20 \%)$ at $40 \mathrm{~kJ} \mathrm{~g}^{-1} \mathrm{CNC}$. Likewise, the hydrodynamic diameter of the particle collective decreased logarithmically from 94.5 to $73.5 \mathrm{~nm}(-22 \%)$ in the same processing window. While the rod length, below which $95 \mathrm{wt} \%$ of the CNCs were found, decreased from 306.5 to $231.8 \mathrm{~nm}$ (-24\%) from 2 to $40 \mathrm{~kJ} \mathrm{~g}^{-1} \mathrm{CNC}$, the shape factor of the main particle fraction ranged from 1.0 to 1.1 , which indicated a decreasing number of dimers and clusters in the particle collective. In summary, progressing ultrasonication caused a shift of the particle length distribution to shorter particle lengths and simultaneously induced narrowing of the distribution. The suspension's electrical conductivity concurrently increased, which has been attributed to faster diffusion of smaller particles and exposure of previously obscured surface charges. Colloidal stability, investigated through electrical AF4 and electrophoretic light scattering, was not affected by 
ultrasonication and, therefore, indicates no de-sulfation by the applied ultrasound treatment. Occurrence of minor $\mathrm{CNC}$ agglomeration at low ultrasound energy densities over the course of 6 months suggest the effect was not unmitigatedly permanent.

Keywords Cellulose nanocrystals (CNCs) . Ultrasound · Ultrasonication - Asymmetrical flow field-flow fractionation (AF4) · Electrical asymmetrical flow field flow fractionation (EAF4) . Particle size distribution (PSD) · Colloidal stability

\section{Introduction}

Cellulose nanocrystals (CNCs) have attracted substantial attention in academia and industry over the past decade. They are considered, through extensive review, to be renewable, biodegradable, and non-toxic high-performance building blocks for numerous potential commercial applications (Habibi et al. 2010; Moon et al. 2011; Abitbol et al. 2016; Vanderfleet and Cranston 2020). CNCs were initially isolated by Nickerson and Habrle (1947) and Rånby and Ribi (1950) from cotton cellulose via acid-catalyzed heterogeneous hydrolysis. Over the years, numerous manufacturing methods were developed (Brinchi et al. 2013; Jonoobi et al. 2015; Reid et al. 2017; Trache et al. 2017) and a number of natural cellulose sources have been successfully employed (Trache et al. 2017). To date, however, both academia and industry have focused on isolation of $\mathrm{CNCs}$ via acid-catalyzed hydrolysis of cotton or wood. The mostly crystalline nanoparticles that remain after heterogeneous hydrolysis - the CNCs - can be approximated as cylindrical, semi-crystalline rods with diameters ranging between 3 and $50 \mathrm{~nm}$ and aspect ratios of 5 to 50 (Souza Lima et al. 2003, ISO 2017b, Lin et al. 2019). Besides chain cleavage, hydrolysis of cellulose with sulfuric acid facilitates sulfation by esterification of some of the hydroxy sites of $\mathrm{CNCs}\left(R-\mathrm{OSO}_{3}{ }^{-}\right)$. The introduced electrical surface charge promotes colloidal stability in polar media due to electrostatic interparticle repulsion.

When the desired extent of hydrolysis of cellulose is approached during CNC production, the reactant solution-now containing CNCs, acid, and byproducts-is quenched with water. Its high ionic strength causes agglomeration of CNCs (Phan-Xuan et al. 2016; Metzger et al. 2020). Peptization and the formation of a stable colloidal suspension is achieved by separating CNCs from acid and byproducts via sedimentation and filtration (Marchessault et al. 1961; Rudie 2017; Gicquel et al. 2019). Purified suspensions are considered long-term stable, however, CNCs tend to form lateral agglomerates and clusters of multiple nanocrystals due to strong interparticle hydrogen bonds (Marchessault et al. 1961; Chen et al. 2020). Further mechanical treatment, such as ultrasonication, is therefore needed to individualize the nanoparticles (Revol et al. 1994; Dong et al. 1998; ISO 2018a). The apparent particle size decreases with increasing energy input as a result of ultrasound treatment (Marchessault et al. 1961; Dong et al. 1998; Jakubek et al. 2018; Mazloumi et al. 2018; Shojaeiarani et al. 2020). For example, Brinkmann et al. (2016) observed a bi-exponential decrease of hydrodynamic apparent particle diameter with increasing ultrasound energy. The applied power (transferred energy per unit time) predominates the effect on particle size rather than absolute energy input or treatment time (Beuguel et al. 2018; Shojaeiarani et al. 2020). The overall impact of ultrasound treatment on particle size distribution (PSD) is assumed to be permanent and cumulative when there is no change of state; for example, through precipitation or drying (Beck et al. 2011, 2012). Typically, brief ultrasonication at an energy density, $e_{U S}$, of $\sim 2 \mathrm{~kJ} \mathrm{~g}^{-1} \mathrm{CNC}$ is sufficient to disperse agglomerated CNCs (Dong et al. 1998; Beck et al. 2012). However, a more recent study suggests that an $e_{U S}$ of $10 \mathrm{~kJ} \mathrm{~g}^{-1} \mathrm{CNC}$ is required to disperse CNCs without any remaining large agglomerates (Beuguel et al. 2018).

To the best of our knowledge, no studies have reported treatment of CNCs with high-frequency ultrasound. In this respect, note that high-frequency ultrasound irradiation of cellulose in water can cause homolytic fission of water and subsequent radical depolymerization of cellulose by scission of glycosidic bonds and by delamination due to hydrogen bond cleavage (Haouache et al. 2020). In contrast, lowfrequency ultrasonic laboratory baths are not sufficiently powerful to individualize CNCs (Beck et al. 2012). On a related note, Beck et al. (2011) showed that the use of conventional ultrasound homogenizers with a frequency of $20 \mathrm{kHz}$ and a power of up to $130 \mathrm{~W}$ facilitates $\mathrm{CNC}$ individualization. These 
process parameters reportedly do not cause breakage of covalent bonds between CNCs and sulfate halfesters-provided that thermal energy, converted from ultrasound energy, is dissipated during the treatment. The reaction rate of autocatalyzed de-sulfation by proton counterions associated with sulfate half-esters significantly increases with temperature (Dong and Gray 1997; Jiang et al. 2010; Beck and Bouchard 2014). In this regard, however, Dong et al. (1998) observed only an insignificant increase of sulfate halfester density on CNC surfaces when temperature was maintained constant, just above $0{ }^{\circ} \mathrm{C}$, during ultrasonication. This increase could be attributed to the gained free surface through particle individualization (Girard et al. 2021). Girard et al. (2021) further noted that geometric and volumetric effects during ultrasonication must be considered and suggested an ultrasonication protocol for improved dispersion efficiency based on a numerical approach.

Common methods for determining CNC size and morphology — or for monitoring changes in PSD — are dynamic light scattering (DLS), atomic force microscopy (AFM), and transmission electron microscopy (TEM) (Foster et al. 2018). These methods are usually performed off-line and come with considerable limitations. Particle sizing by DLS is performed under the assumption that the sample comprises particles having a single, constant rate of diffusion. Rod-like nanoparticles like CNCs, however, have different translational diffusion constants parallel and perpendicular to the particle axis (Souza Lima et al. 2003). Thus, the hydrodynamic apparent particle size obtained from DLS cannot be directly correlated to actual particle size. Furthermore, as an intensity-based method, DLS results may be dominated by the presence of agglomerates and particle clusters (Chen et al. 2020). Regardless, DLS is an established semi-quantitative method for analyzing dispersed, rod-like CNCs (Fraschini et al. 2014; Foster et al. 2018). Imaging techniques, such as AFM and TEM, enable the full quantitative description of CNC size and morphology. However, both techniques are preparatorily challenging and require sufficiently large data sets to adequately describe PSD (Jakubek et al. 2018; Campano et al. 2021). Native size and morphology of CNCs in a liquid suspension may significantly differ from the dry CNCs required for AFM and TEM (Guan et al. 2012). Furthermore, TEM imaging commonly involves negative staining of CNCs with uranyl acetate, which favors lateral particle agglomeration and generates radioactive waste (Kaushik et al. 2014). Jakubek et al. (2018) therefore suggested the use of asymmetrical flow field-flow fractionation (AF4) for the analysis of a full distribution of dispersed CNCs (Supporting Information, Fig. S1). Among the field-flow fractionation techniques, which are subdivided by the applied separation force, AF4 is the most prominent subtechnique (Contado 2017; Drexel et al. 2020a, b).

Guan et al. (2012) were the first to apply AF4 in combination with multi-angle light scattering (MALS) to investigate the separation of CNCs. They established a rod-like form factor and found that the length distribution of CNCs calculated from MALS data agrees well with the length from TEM analyses of early eluting fractions. Espinosa et al. (2017) and Ruiz-Palomero et al. (2017) used AF4-MALS for qualitative detection of CNCs and correlated their results with DLS. While Espinosa et al. (2017) demonstrated the ability to display small particle fractions that were not resolvable with DLS, RuizPalomero et al. (2017) used AF4-MALS for detecting CNCs extracted from consumer goods. More recently, Mukherjee and Hackley (2018) focused on the improvement of AF4, in combination with MALS and differential refractometry (dRI), for size-based separation and on-line characterization of CNCs. However, their calculated mean rod length of $146 \mathrm{~nm}$ differed from reported mean lengths of the same CNC sample calculated from TEM images $(87 \mathrm{~nm})$ and AFM images $(76 \mathrm{~nm})$ by Jakubek et al. (2018). In this regard, Chen et al. (2020) focused on surface imaging analysis of single CNC fractions previously separated and analyzed using multi-detector AF4. While early eluting fractions contained mostly individual CNCs, they predominantly detected clustered $\mathrm{CNCs}$ at higher retention times-even though the suspension was ultrasonicated at $5 \mathrm{~kJ} \mathrm{~g}^{-1}$ CNC beforehand.

Thus far, only a handful of studies have applied AF4 to CNCs. These are summarized in Supporting Information, Table $\mathrm{S} 1$ and validate multi-detector AF4 for semi-quantitative and quantitative analysis of CNC suspensions. It is reported that, even in ultrasonicated and stable CNC suspensions, a considerable particle fraction exists in the agglomerated state, which implies a broad PSD. However, a narrow PSD of CNCs facilitates better dispersibility and reinforcement capacity in polymer matrices (Chen et al. 2020; 
Shojaeiarani et al. 2020), promotes tailoring of selfassembly properties in the liquid crystalline phase and in chiral nematic films (Dong et al. 1998; Beck et al. 2011; Gicquel et al. 2019), and enables control over the viscosity of CNC suspensions (Shafiei-Sabet et al. 2012; Beuguel et al. 2018; Gicquel et al. 2019). Furthermore, a narrower PSD may be useful in nanotoxicological studies (Roman 2015; Shatkin and Kim 2015) and for in-line detection of CNCs and monitoring their PSD in complex environments (Foster et al. 2018).

The aforementioned studies on the impact of ultrasonication on CNC suspensions showed that the ultrasound treatment facilitates dispersion of clusters and narrowing of the PSD. However, while assessing their state of agglomeration with DLS, AFM, and TEM is qualitatively feasible, this comes with abovementioned shortcomings. In this context, our primary objective was the targeted dispersion of CNC clusters by ultrasonication and the investigation of its impact on the full distribution of species via multi-detector AF4 and off-line techniques. Anticipated effects of ultrasonication on colloidal CNCs are summarized in Fig. 1.

Our hypotheses were based on reported literature that (1) ultrasonication induces breakage of agglomerates and clusters. An increase of the applied ultrasound energy density is generally associated with a cumulative reduction of cluster size and, thus, a decrease of mean particle size (Fig. 1a). This may go unnoticed with commonly applied off-line imaging techniques, such as AFM and TEM, because laterally cohering CNCs may fabricate similar particle lengths and diameters compared to individual particles in the initial suspension. Therefore, it does not necessarily significantly change the apparent PSD of a sample (Jakubek et al. 2018). However, surface charges obscured inside the clusters contribute to the net charge after ultrasonication (Girard et al. 2021). Moreover, (2) CNC ultrasonication in water may induce the release of charged moieties from the boundwater surface layer of CNCs (Fig. 1b) (Bouchard et al. 2016; Beuguel et al. 2018). In this context, Beck et al. (2011) and Bouchard et al. (2016) attributed the effect of ultrasonication on rheological and self-assembly properties of CNC suspensions mainly to a change of electrostatic particle interactions. No de-sulfation has been reported during ultrasonication of sulfated, $\mathrm{Na}^{+}$exchanged $\mathrm{CNCs}$ at room temperature and ultrasound energy density of $10 \mathrm{~kJ} \mathrm{~g}^{-1} \mathrm{CNC}$ (Beuguel et al. 2018). We, however, investigated the effect of ultrasonication on protonated CNCs for which autocatalyzed de-sulfation has already been reported at low temperatures (Fig. 1c) (Jiang et al. 2010; Beck and Bouchard 2014). Samples were, therefore, kept at $2{ }^{\circ} \mathrm{C}$ during all ultrasonication experiments to mitigate potential de-sulfation; nevertheless, ultrasound induces cavitation and local temperature peaks occur. Furthermore, (3) deagglomeration by ultrasonication is permanent and cumulative when there is no change of state (Beck et al. 2011, 2012).

Expanded understanding of the impact of ultrasonication on $\mathrm{CNC}$ suspensions provides potential for tailoring the PSD of CNCs through breakage of particle clusters. This is also emphasized by the ongoing development of technical specification ISO/ TC 229-PWI 23151 (ISO 2018a), which addresses ultrasound-assisted deagglomeration of redispersed sulfated CNCs.

\section{Materials and methods}

Materials

Whatman ashless filter aids (cotton $\alpha$-cellulose) were purchased from Sigma-Aldrich (Taufkirchen, Germany). Sulfuric acid $\left(\mathrm{H}_{2} \mathrm{SO}_{4}, 96 \%\right)$ was used for preparing CNCs and was purchased from Carl Roth (Karlsruhe, Germany). The isolation of $\mathrm{CNCs}$ was performed under nitrogen atmosphere $\left(\mathrm{N}_{2}, 99.8 \%\right.$, Linde, Pullach, Germany). Sodium chloride ( $\mathrm{NaCl}$, $99.5 \%$ ) and sodium hydroxide $(\mathrm{NaOH}(\mathrm{aq}), 2 \mathrm{mM})$, required for conductometric titration, were purchased from Carl Roth. Protonated strong acid cation ionexchange resin (SAC; Dowex Marathon C) and Amberlite MB6113 $\mathrm{H}^{+} \mathrm{OH}^{-}$mixed bed ion-exchange resin $(\mathrm{MB})$ were purchased from Sigma-Aldrich and thoroughly rinsed with water before use. Syringe filters with hydrophilic polyethersulfone (PES) membranes and a pore size of $0.45 \mu \mathrm{m}$ (CHROMAFIL Xtra PES) and hydrophilic glass fiber membranes with a pore size of $1 \mu \mathrm{m}$ (CHROMAFIL Xtra GF) were purchased from Macherey-Nagel (Düren, Germany) and used for filtration of CNC suspensions. Sodium chloride ( $\mathrm{NaCl}, 99.5 \%$, Avantor Performance Materials, Gliwice, Poland) was diluted to $1 \mathrm{mM}$ and used as the eluent in $\mathrm{AF} 4$ for $\mathrm{CNC}$ fractionation. All eluents 


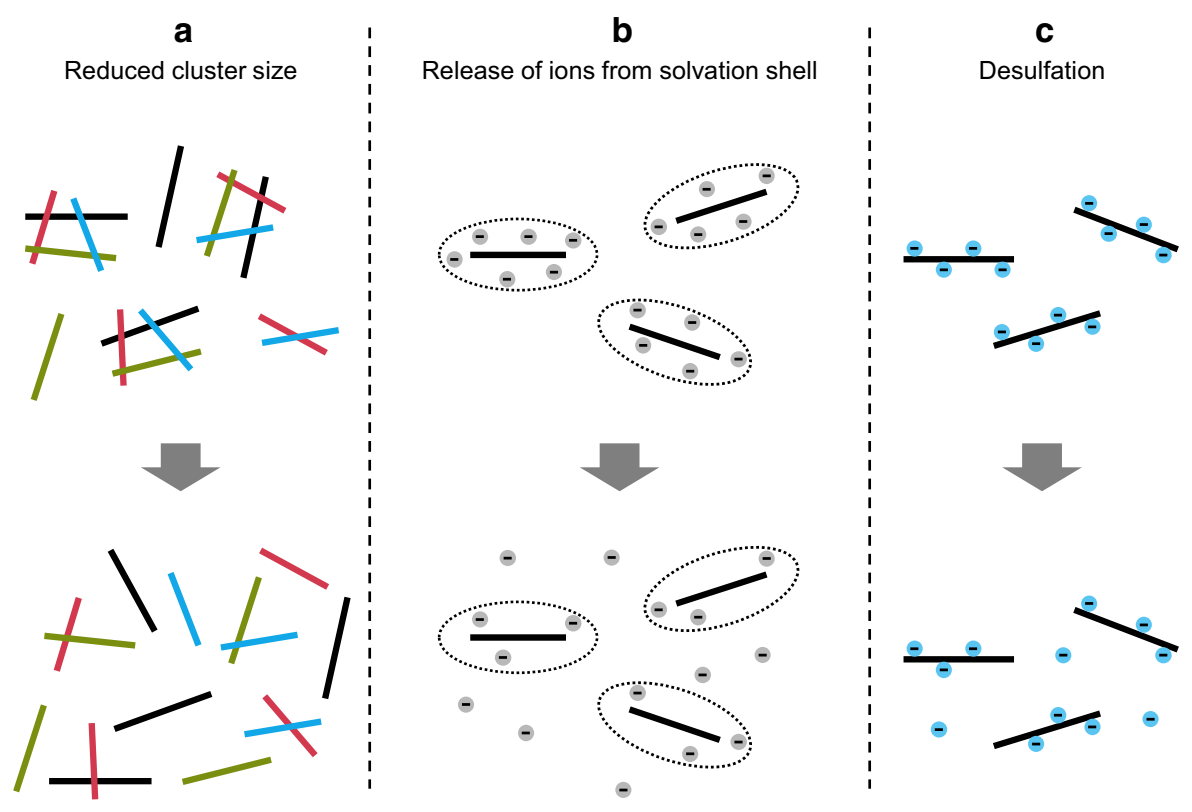

Fig. 1 Effects potentially involved in the ultrasound treatment of colloidal CNCs

were filtered by vacuum using a membrane filter with a pore size of $0.1 \mu \mathrm{m}$ (Durapore, Merck, Tullagreen, Ireland).

All chemicals were used in their as-received state. Ultrapure (type 1) water $\left(\mathrm{H}_{2} \mathrm{O}\right)$ with a resistivity of $18.2 \mathrm{M} \Omega \mathrm{cm}$ (Milli-Q Direct 8 system, Merck Chemicals, Schwalbach, Germany) was used to prepare solutions for all experiments.

\section{Preparation of $\mathrm{CNCs}$}

Colloidal suspensions of sulfated CNCs were prepared through the heterogeneous hydrolysis of cellulose with sulfuric acid, based on the method of Cranston and Gray (2006) and Metzger et al. (2020). Cotton cellulose was dried at $105{ }^{\circ} \mathrm{C}$ for $30 \mathrm{~min}$ to remove adsorbed water. Meanwhile, $96 \mathrm{wt} \%$ sulfuric acid was diluted to $64.2 \mathrm{wt} \%$ with water and pre-heated to $45{ }^{\circ} \mathrm{C}$ in a stirred tank reactor equipped with an anchor-type stirrer (Atlas, Syrris, Royston, UK). The dried cellulose was then added in at a mass-mixing ratio of $10 m_{\mathrm{H}_{2} \mathrm{SO}_{4}} m_{\text {cellulose }}^{-1}$ with the weighed portions of sulfuric acid, $m_{\mathrm{H}_{2} \mathrm{SO}_{4}}$, and cellulose, $m_{\text {cellulose }}$; the reactant solution was stirred constantly at $200 \mathrm{rpm}$ under nitrogen atmosphere. The reaction was quenched by ten-fold dilution with $4{ }^{\circ} \mathrm{C}$ water after
$45 \mathrm{~min}$ and stirring was continued at $12{ }^{\circ} \mathrm{C}$ for $30 \mathrm{~min}$.

Excess acid and soluble byproducts were decanted, after leaving the precipitate to settle overnight. The resultant cloudy suspension was washed by two-fold centrifugation for $15 \mathrm{~min}$ at a relative centrifugal force of $4250 \times \mathrm{g}$ (Centrifuge $5910 \mathrm{R}$, Eppendorf, Hamburg, Germany), followed by decanting and further dilution with water. The precipitate was filled into regenerated cellulose tubes with a molecular weight cutoff of 12-14 kDa (ZelluTrans/ROTH T3, Carl Roth, Karlsruhe, Germany) and remaining acid and soluble byproducts were removed by dialysis against running water $\left(0.5 \mathrm{~L} \mathrm{~h}^{-1}\right)$ at $23{ }^{\circ} \mathrm{C}$ in a $7 \mathrm{~L}$ glass reactor for 10 days. Agglomerates were broken via ultrasonication using a homogenizer (Sonopuls HD 3400 with the sonotrode VS $70 \mathrm{~T}$, Bandelin, Berlin, Germany) at a specific energy of $2 \mathrm{~kJ} \mathrm{~g}^{-1}$ cellulose and a power of $33.3 \mathrm{~W}$ in a cooled secondary glass container at $2{ }^{\circ} \mathrm{C}$. Incompletely hydrolyzed solid cellulosic residues were then removed by centrifugation for $15 \mathrm{~min}$ at $4250 \times \mathrm{g}$. The final stock suspension of never dried sulfated and protonated CNCs was stored in a sealed container at $4{ }^{\circ} \mathrm{C}$ until further use. 
Ultrasound treatment of CNCs

The CNC stock suspension was diluted to a particle concentration of $1 \mathrm{wt} \%$. The abovementioned homogenizer was used for ultrasound treatment and the probe was adjusted according to ISO/CD TS 23151 (2018a) to ensure minimal bubbling and aerosoling. The suspension was placed in a temperature-controlled secondary glass container at $2{ }^{\circ} \mathrm{C}$. The sample temperature was constantly monitored and treatment was paused when the temperature exceeded $4{ }^{\circ} \mathrm{C}$. The ultrasound power was set to $33.3 \mathrm{~W}$. Aliquots were extracted from the stock suspension at seven prespecified energy inputs and the remaining suspension was further treated until an energy input of $40 \mathrm{~kJ} \mathrm{~g}^{-1} \mathrm{CNC}$ was reached (Supporting Information, Table S2).

Removal of ionic species by ion-exchange resins

Ultrasonicated aliquots were split and one respective half was stored at $4{ }^{\circ} \mathrm{C}$. Ionic species were then removed from the other halves by successive batch treatments with MB followed by SAC as suggested by Abitbol et al. (2013). Resins were added in at a resinto-CNC mass ratio of eight and the aliquots were continuously stirred for $24 \mathrm{~h}$. After each treatment, the aliquots were rinsed with water over a sieve and filtered with $0.45 \mu \mathrm{m}$ syringe filters before they were stored at $4{ }^{\circ} \mathrm{C}$. No loss of CNCs was assumed by using microporous resins (Beck et al. 2015).

\section{CNC morphology}

Chen et al. (2020) separated sulfated, $\mathrm{Na}^{+}$-exchanged CNCs from softwood pulp with AF4 and classified them, with the help of imaging techniques, as singles, dimers, and clusters. They predominantly detected singles and dimers in early eluting fractions and the relative proportion of clusters in the particle collective increased toward later fractions. Herein, we assume that sulfated, protonated CNCs from cotton are composed of similar nano-objects and nanostructures and refer to them according to the illustration in Fig. 2.

Sample naming

CNC suspensions are named here by the applied ultrasound energy density. For example: CNC-2 for an input of $2 \mathrm{~kJ} \mathrm{~g}^{-1} \mathrm{CNC}$ and $\mathrm{CNC}-40$ for $40 \mathrm{~kJ} \mathrm{~g}^{-1}$

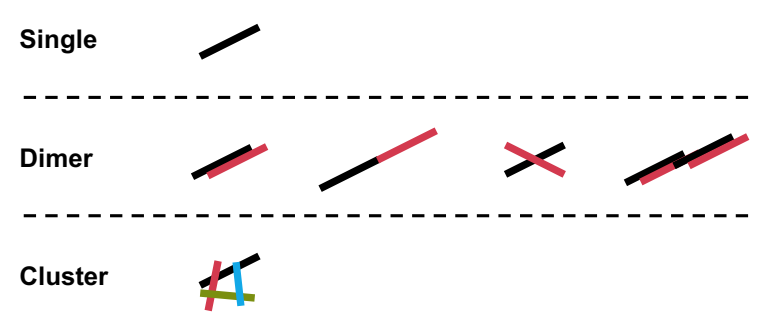

Fig. 2 Classification of CNCs, based on the work of Chen et al. (2020)

CNC. Aliquots treated with ion-exchange resins are further denoted with a postposed "R". For example: CNC-2-R for CNC-2 treated with ion-exchange resin.

\section{Fractionation of CNCs by AF4}

Size-based fractionation of colloidal CNCs was performed at $25^{\circ} \mathrm{C}$ using an $\mathrm{AF} 4$ system (AF2000) equipped with an autosampler (PN5300) and a channel thermostat (PN4020). All experiments were monitored using on-line coupled MALS and ultraviolet spectroscopy (UV) detectors. The CNC suspensions were filtered with $1 \mu \mathrm{m}$ syringe filters and then diluted to $0.04 \mathrm{wt} \%$. Sample volumes of $20 \mu \mathrm{L}$ were injected using $1 \mathrm{mM} \mathrm{NaCl}$ as the eluent. The applied separation parameters are shown in Table 1.

The planar separation channel (Supporting Information, Fig. S1) had a tip-to-tip length of $277 \mathrm{~mm}$ and was equipped with a Mylar spacer with a thickness of $350 \mu \mathrm{m}$ and a regenerated cellulose (RC) membrane with a molecular weight cutoff of $10 \mathrm{kDa}$. Using the slot outlet option of the AF4 channel (PN1650), the eluting channel flow was split into a detector flow with a CNC-enriched fraction at a flow rate of $0.30 \mathrm{~mL} \mathrm{~min}^{-1}$ and a particle-free eluent fraction at a flow rate of $0.20 \mathrm{~mL} \mathrm{~min}{ }^{-1}$, which was discarded. All instruments and consumables in this section that were not mentioned in earlier sections were provided by Postnova Analytics GmbH (Landsberg am Lech, Germany).

Instrument measurements

Preliminary evaluations of CNC suspensions separated with $\mathrm{AF} 4$ showed high repeatability and reproducibility in accordance with the guidelines of ISO/TS 21362 (ISO 2018b). Thus, fractionation and related measurements were replicated twice. All other 
Table 1 Separation parameters for AF4

\begin{tabular}{lllll}
\hline Step & Flow type and rate $\left(\mathrm{mL} \mathrm{min}^{-1}\right)$ & Duration $(\mathrm{min})$ & Mode \\
\hline General & Detector flow & 0.30 & & \\
Focusing and injection & Slot flow & 0.20 & & \\
& Injection flow & 0.20 & 6 & \\
& Cross flow & 1.00 & & \\
Transition & Focus flow & 1.30 & & Constant \\
Elution 1 & & & 0.5 & Power decay (exponent 0.2) \\
Elution 2 & Cross flow & 1.00 & 2 & Constant \\
Elution 3 & Cross flow & 1.00 & 40 & \\
Rinse & Cross flow & 0.10 & 40 & 15 \\
\hline
\end{tabular}

measurements were performed at least in triplicate and are presented with the $95 \%$ confidence interval of the mean.

\section{Optical measurements}

Multi-angle light scattering The scattered light intensity of fractionated CNCs was detected under 19 active angles ranging from $12^{\circ}$ to $156^{\circ}$ with an on-line coupled MALS detector PN3621 (Postnova) at a wavelength of $532 \mathrm{~nm}$ and a cell temperature of $35{ }^{\circ} \mathrm{C}$. The angular-dependent scattering intensity function, $P(\theta)$, is given by

$P(\theta)=\frac{2}{q L} \operatorname{Si}(q L)-\left(\frac{\sin \frac{q L}{2}}{\frac{q L}{2}}\right)^{2}$,

with scattering angle, $\theta$; rod length, $L$; scattering vector, $q=4 \pi n_{0} \sin \left(\frac{\theta}{2}\right) / \lambda$; and sine integral function, $\operatorname{Si}(q L)$ (van de Hulst 1958). $q$ includes the refractive index of the eluent, $n_{0}$, and the incidental wavelength, $\lambda$. All data were normalized with respect to the signal at an angle of $90^{\circ}$. Standard polystyrene beads with a nominal size of $60 \mathrm{~nm}$ (NIST 2021) were fractionated and used to normalize detectors at different angles with respect to $90^{\circ}$ to the radius of gyration, $r_{g}$, as a function of retention time by considering the angulardependent spherical scattering intensity function $P(\theta)$ (Supporting Information, Fig. S2). In accordance with Mukherjee and Hackley (2018), a rod model was best suited for fitting MALS data of fractionated CNCs. Consequently, the relationship of $r_{g}$ and $L$ for thin rigid rods with a high $L-d$ ratio, where $d$ is the particle diameter, can be approximated as (Stepto et al. 2015; Mukherjee and Hackley 2018):

$r_{g}^{2}=\frac{L^{2}}{12} \quad$ for $d \ll L$

The evaluation of $r_{g}$ was performed using the NovaMALS software (Postnova Analytics GmbH 2020c). In addition, the hydrodynamic radius, $r_{h}$, which depends on the translational diffusion coefficient of the CNCs, was derived from AF4 conditions, such as cross flow rate, channel thickness, and channel flow rate (Wahlund and Giddings 1987; Litzen and Wahlund 1991). The effective channel height was determined from the retention times of fractionated standard polystyrene beads, with a nominal diameter of $60 \mathrm{~nm}$ (NIST 2021). $r_{h}$ as a function of retention time was evaluated using the NovaAnalysis software (Postnova Analytics GmbH 2020a).

Ultraviolet spectroscopy An UV detector (PN3211, Postnova) was coupled on-line with AF4 and absorbance was recorded at a wavelength of $254 \mathrm{~nm}$. The analyte recovery, $R e c$, was evaluated from the peak area of the eluted sample, $A_{S}$, and the peak area of the sample measured in absence of any separation field, $A_{D}$, according to ISO/TS 21362 (ISO 2018b):

$\operatorname{Rec}(\%)=\frac{A_{S}}{A_{D}} \cdot 100 \%$.

Zeta potential ( $\zeta$ ) EAF4 (EAF2000, Postnova) was used to determine the size-resolved electrophoretic mobility and zeta potential $(\zeta)$ of colloidal CNCs online. The same fractionation parameters as those for the size-based fractionation experiment with AF4 
were applied (Table 1). Here, however, an amphiphilic RC membrane (Postnova) with a molecular weight cutoff of $10 \mathrm{kDa}$ was used. The cross flow was superimposed with an electrical field (PN2410), which was controlled with the NovaFFF software (Postnova Analytics GmbH 2020b). EAF4 data were evaluated with the NovaAnalysis software (Postnova Analytics GmbH 2020a).

Off-line zeta potential measurements were performed with a Zetasizer Nano ZSP (Malvern Instruments, Worcestershire, UK) in folded capillary cells (DTS1070). CNC dispersions at a concentration of $0.25 \mathrm{wt} \%$ were analyzed after equilibration for $30 \mathrm{~min}$ at $25{ }^{\circ} \mathrm{C}$.

For both on-line and off-line measurements $\zeta$ is calculated from the electrophoretic mobility assuming Smoluchowski behavior with $f(\kappa r)=1.5$ for Henry's function, where $\kappa$ is the Debye length and $r$ the particle radius (Smoluchowski 1906).

Dynamic light scattering The hydrodynamic apparent particle diameter, $d_{h}$, was determined off-line from DLS using a Zetasizer Nano ZSP under a backscatter detection angle of $173^{\circ} . d_{h}$ and the dispersity, $Ð$, from cumulants analysis was obtained according to ISO 22412 (ISO 2017a) for $0.04 \mathrm{wt} \% \mathrm{CNC}$ suspensions after equilibration for $3 \mathrm{~min}$ at $25{ }^{\circ} \mathrm{C}$ in disposable polystyrene cuvettes (Stepto 2009).

\section{Conductometric titration}

The surface sulfate half-ester concentration, $c_{S}$, of colloidal CNCs was determined by conductometric titration, as suggested by Beck et al. (2015). For the electrical conductivity measurements, $5 \mathrm{~mL}$ of a 1 $\mathrm{wt} \% \mathrm{CNC}$ dispersion was diluted into $80 \mathrm{~mL}$ of water. To elevate the electrical conductivity, $\sigma$, to a measurable level, $1 \mathrm{~mL}$ of $0.1 \mathrm{M} \mathrm{NaCl}$ (aq) was added. The titrant used was $2 \mathrm{mM} \mathrm{NaOH}$ (aq) in $0.2 \mathrm{~mL}$ increments under constant stirring. Stable conductivity readings (Konduktometer 703 with the electrode sensor SE 204, Knick, Berlin, Germany) were recorded 30-60 s after each addition and plotted against the amount of $\mathrm{NaOH}$ added. The equivalent amount of $\mathrm{NaOH}$, and, thus, the concentration of accessible sulfate half-ester groups of the CNCs, was calculated from the intersect of the least squares regression lines of the two linear branches $\left(R^{2} \approx 1\right)$ of the conductivity curve. All measurements were performed at $25{ }^{\circ} \mathrm{C}$.

\section{Results and discussion}

Size distributions of ultrasonicated CNCs

\section{Effect of ultrasonication on size distribution}

Suspensions of $1 \mathrm{wt} \%$ colloidal CNCs were ultrasonicated with increasing energy densities ranging from 2 to $40 \mathrm{~kJ} \mathrm{~g}^{-1} \mathrm{CNC}$. Each sample was then size fractionated by AF4. The fractograms in Fig. 3a show MALS intensities at $90^{\circ}$ (solid lines) along with the radii of gyration of three selected samples (filled points). As shown in Fig. $3 b$, the UV absorbance signals were slightly left-shifted in regard to the MALS scattering intensity. All intensity distributions followed lognormal behavior, in accordance with the AF4 separation pattern, and had peaks, $I_{\max }$, at $t_{R}\left(I_{\max }\right) . t_{R}\left(I_{\max }\right)$ shifted to shorter retention times with increasing ultrasound energy density, which is equivalent to a shift to smaller particle sizes. Concurrent narrowing of the MALS scattering intensity implied narrowing of the CNC size distribution. Simultaneously, an exponential decrease of $I_{\max }$ with increasing ultrasound energy was observed (Supporting Information, Fig. S3) and the mass-weighted particle concentration, derived from the UV signal, decreased by $25 \mathrm{wt} \%$ from CNC-2 to CNC-40. As all samples were branched off from the same stock solution with constant $\mathrm{CNC}$ concentration, the origin of the apparent mass loss is ambiguous. Throughout all AF4 experiments, the initial void peak remained at a constant and insignificant level. Similarly, no significant field-off peak was observed during rinsing when cross flow was zero, which therefore indicated inconsiderable agglomeration during fractionation. Simultaneously, recoveries of 84-92\% were achieved for all samples while no dependence of $\operatorname{Rec}$ on $e_{U S}$ was observable. Hence, only marginal particle adsorption to or diffusion through the membrane occurred and good separation performance was achieved, in accordance with ISO/TS 21362 (ISO 2018b). No sedimentation was observed for all samples over the course of 1 month and in contrast to UV spectroscopy, conformable count rates during off-line DLS measurements implied no sample loss. The apparent mass loss 


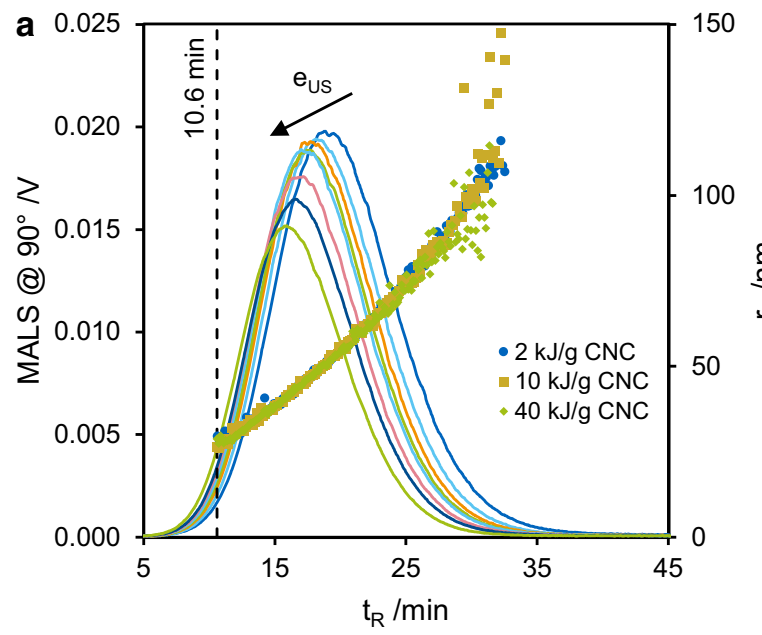

Fig. 3 Fractograms of $\mathrm{CNC}$ suspensions ultrasonicated at different energy densities ranging from 2 to $40 \mathrm{~kJ} \mathrm{~g}^{-1} \mathrm{CNC}$. a MALS scattering intensity distributions and radii of gyration are represented by solid lines and filled points, respectively.

with increasing ultrasound energy input revealed by the UV detector may result from an additional size and shape-dependent scattering contribution of rod-like CNCs and irregularly shaped clusters to the overall UV absorbance signal (van de Hulst 1958). This may lead to overestimation of the overall CNC concentration and, hence, UV spectroscopy is not suited to evaluate the mass balance at varying ultrasound energy input. Consequently, the comparability of incremental size fractions decreases with increasing retention time and quantification is only feasible for early eluting fractions, wherein singles and dimers predominate (Chen et al. 2020).

Figure 4a shows the shift of $t_{R}\left(I_{\max }\right)$, as well as the narrowing of MALS intensity distributions, with regard to their full widths at half maximum, FWHM, in response to increasing ultrasound energy density. Both had a linear interrelationship (Fig. 4b) and could be individually approximated logarithmically.

Equivalent to the MALS peak shift in Fig. $4 \mathrm{a}, r_{g}$ at $t_{R}\left(I_{\max }\right)$ and the associated decrease of rod lengths with more extensive ultrasonication followed logarithmic laws. A decrease in $r_{g}\left(I_{\max }\right)$, from $51.4 \pm 0.5$ to $40.9 \pm 0.2 \mathrm{~nm}(-20 \%)$, was observed from CNC2 to CNC-40, and $L$ decreased conformably, from $178.1 \pm 1.7$ to $141.7 \pm 0.7 \mathrm{~nm}$ (Fig. 5). In the same interval, the mean hydrodynamic apparent diameter of the full particle collective, determined by off-line DLS, decreased from $94.5 \pm 0.3$ to $73.5 \pm 0.4 \mathrm{~nm}$

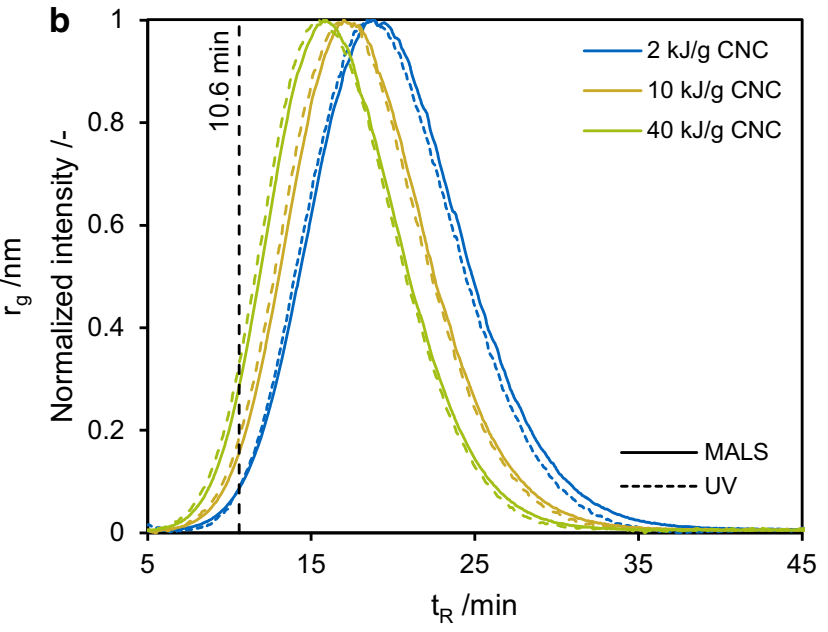

Radii of gyration are displayed for retention times higher than 10.6 min (dashed vertical lines). b UV absorbance signal (dashed lines) are slightly left-shifted with regard to the MALS scattering intensity

$(-22 \%)$. No statistically significant trend was found for $\mathrm{Đ}$, which ranged between moderate values of $0.155 \pm 0.014$ and $0.174 \pm 0.013$ for all samples (ISO 2017a).

It is recognizable from Fig. 3 that in all CNC suspensions notable sample fractions were eluted at retention times earlier than $10.6 \mathrm{~min}$, which, therefore, had an $r_{g}$ below $25 \mathrm{~nm}$. The share of this particular fraction, $\varphi_{<10.6 \mathrm{~min}}$, of the cumulative particle mass was quantified from the UV absorbance signal (Fig. 3b); it increased from $1.0 \pm 0.3$ to $5.3 \pm 0.1 \mathrm{wt} \%$ from CNC-2 to CNC-40, following a power law (Fig. 6a). Consequently, extensive ultrasonication caused progressive dispersion of dimers and clusters and the amount of CNCs with lengths $<$ $87 \mathrm{~nm}$ increased. It is unclear whether this fraction was only comprised of individual CNCs. Reported mean particle lengths of CNCs from cotton $\alpha$-cellulose are typically in the range of 100-250 nm and may vary with hydrolysis severity (Araki et al. 2001; BeckCandanedo et al. 2005; Wang et al. 2012; Boluk and Danumah 2014). Other groups who have analyzed size-fractionated CNC suspensions by MALS in combination with imaging techniques have also not identified particle fractions with lengths $<80 \mathrm{~nm}$ (Guan et al. 2012; Mukherjee and Hackley 2018; Chen et al. 2020). However, all of those studies have only applied a maximal ultrasound density of $5 \mathrm{~kJ} \mathrm{~g}^{-1}$ $\mathrm{CNC}$. Therefore, extensive ultrasonication may induce 

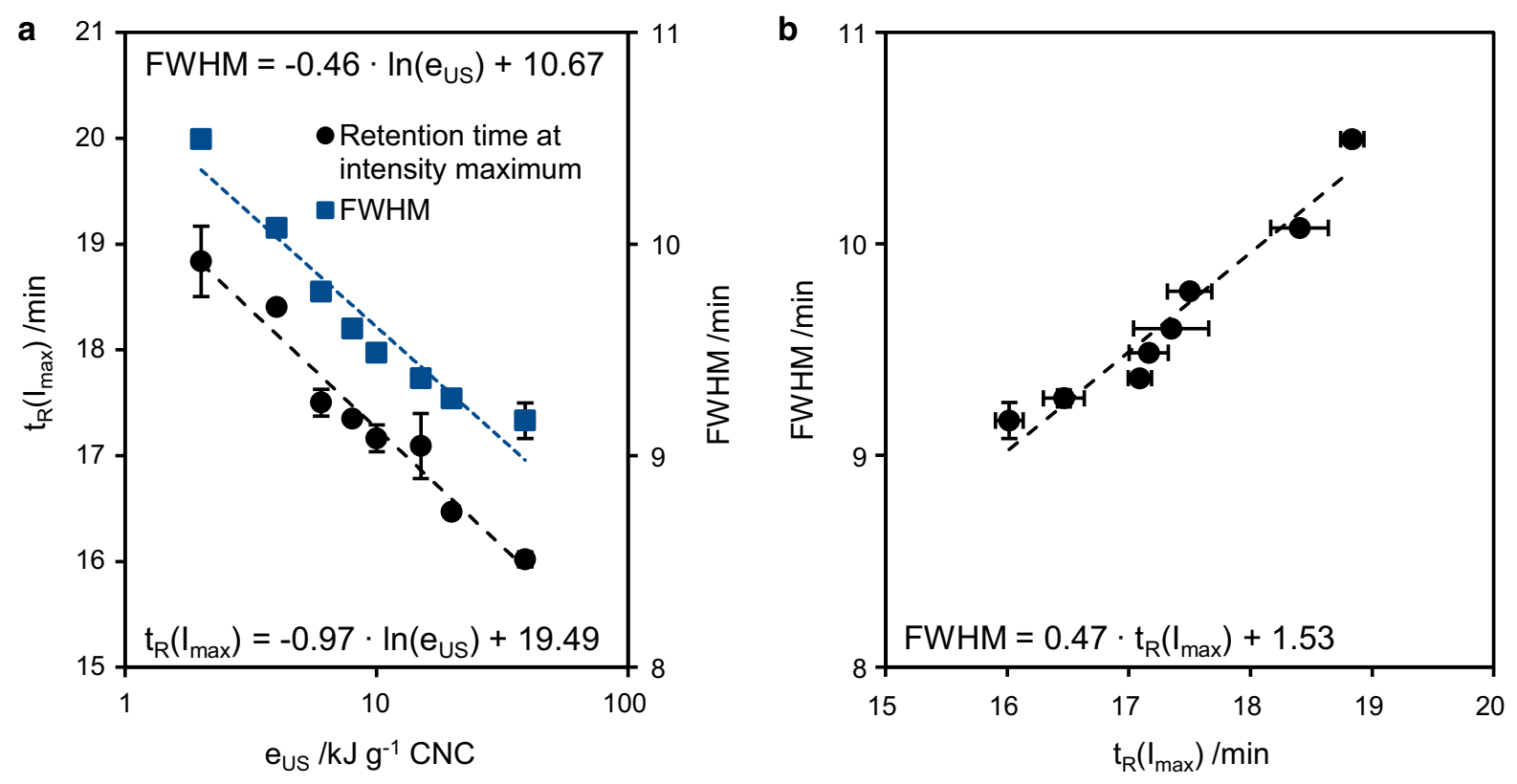

Fig. 4 a Evolution of peak maximum (left vertical axis) and $F W H M$ (right vertical axis) of the MALS signal as a function of ultrasonication energy density and $\mathbf{b}$ the respective relationship of $F W H M$ and peak maximum

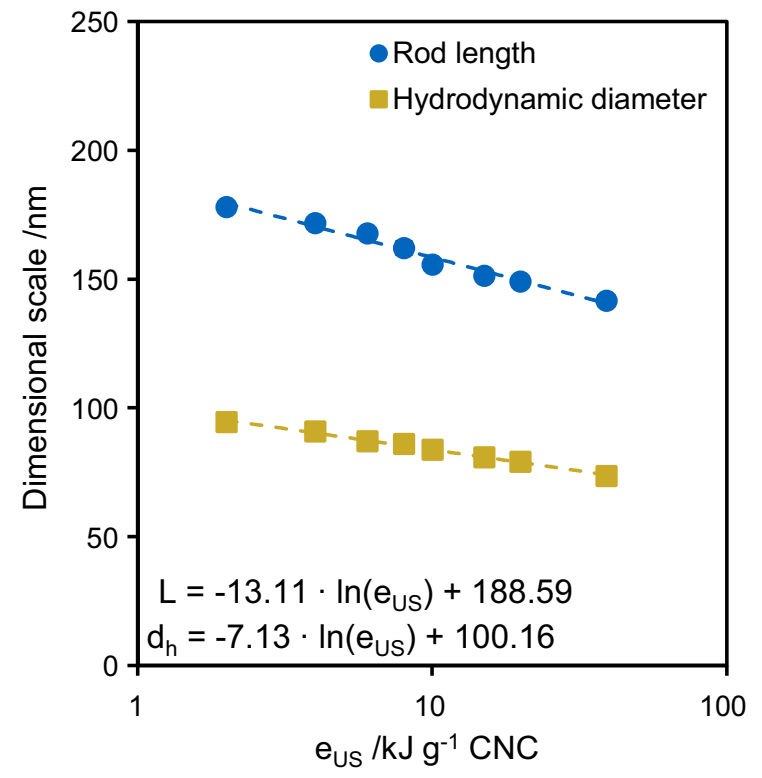

Fig. 5 Rod length $L$ at $I_{\max }$, from AF4-MALS, and hydrodynamic diameter $d_{h}$, from off-line DLS, decrease logarithmically with increasing ultrasound energy. $r_{g}$ and $L$ at $I_{\max }$ are linearly proportional, according to Eq. (2)

a progressing release of not fully immobilized oligosaccharides that were previously trapped in the solvation shells of the particles (Bouchard et al. 2016) or debris from formerly fringed polymer chains that seceded from the bulk particles (Kontturi 2018). Furthermore, acid-catalyzed hydrolysis of cellulose in combination with ultrasonication reportedly enables the production of spherical cellulose nanoobjects with diameters down to $10 \mathrm{~nm}$ (Wang et al. 2007, 2008; Zianor Azrina et al. 2017). Thus, cavitation-induced temperature increase may have induced progressing autocatalysis during ultrasonication of protonated $\mathrm{CNCs}$, facilitating their disintegration into spherical fragments with radii of gyration $<25 \mathrm{~nm}$.

\section{Dispersion of clusters}

The concurrently observed dispersion of large clusters further manifested narrowing of PSD. The rod length, below which $95 \mathrm{wt} \%$ of the CNCs were found, decreased from $306.5 \pm 7.3$ to $231.8 \pm 0.0 \mathrm{~nm}$ (-24\%) from CNC-2 to CNC-40 (Fig. 6b). The concurrent change in shape of clusters was quantifiable by the shape factor, which is represented by the ratio of the radius of gyration and the hydrodynamic radius, $r_{g} r_{h}^{-1}$, that is shown in Fig. 7 . The mass-related large portion of CNCs with lengths of 130-270 nm had shape factors ranging from 1.0 to 1.1 , which increased toward shorter lengths. This substantiates 

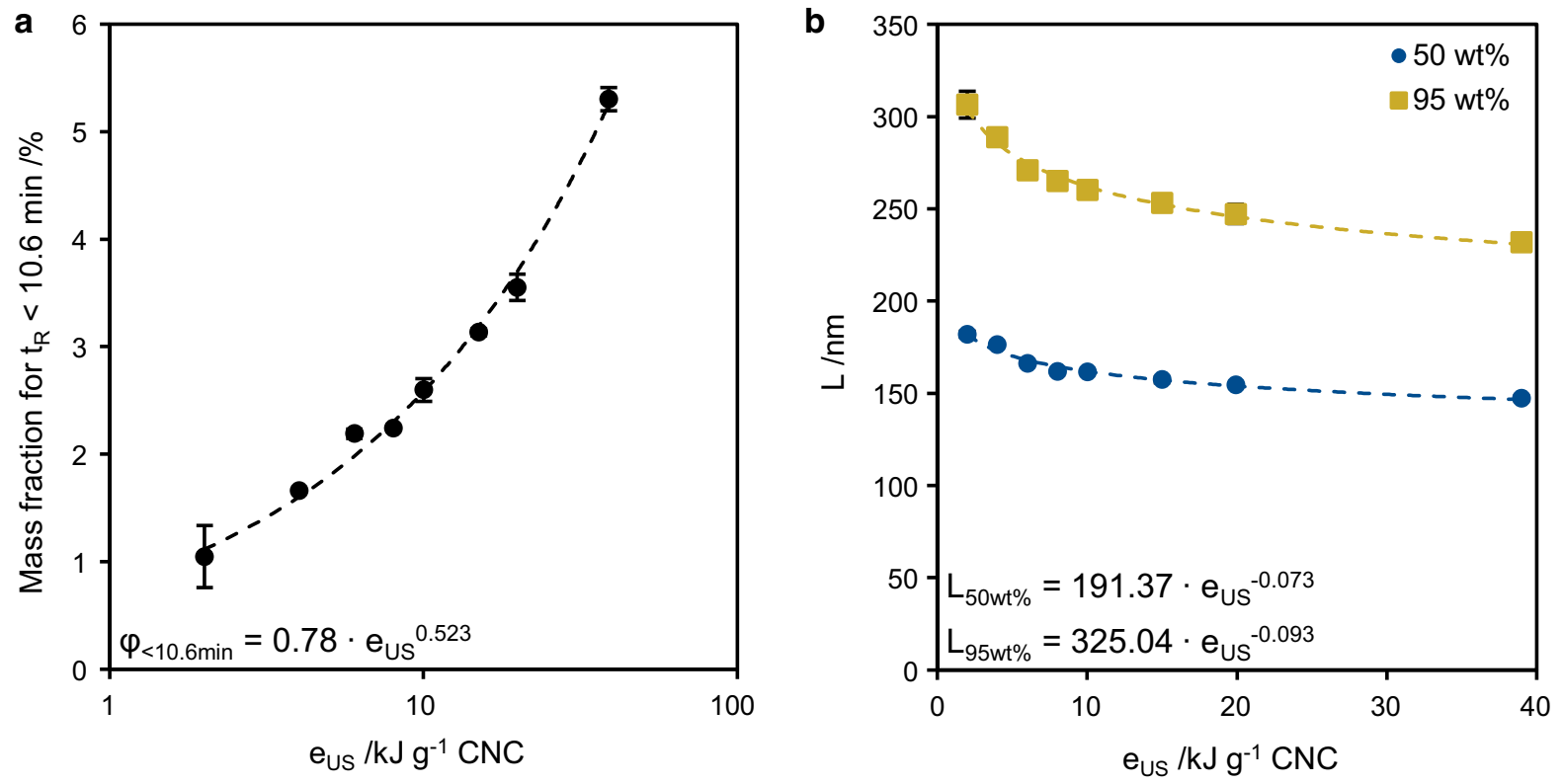

Fig. 6 a Cumulative mass fraction at retention times $<10.6 \mathrm{~min}$, derived from UV signal, and b rod lengths when $50 \mathrm{wt} \%$ and $95 \mathrm{wt} \%$ of CNCs are shorter than this value

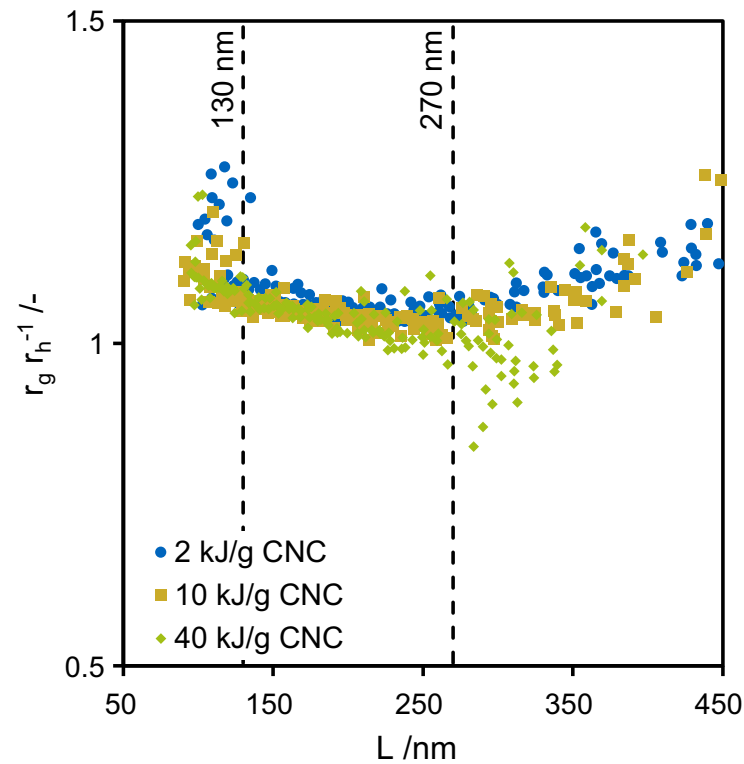

Fig. 7 The shape factor of CNC suspensions as a function of rod length

the observation of Chen et al. (2020) that the number of dimers and clusters decreases toward shorter retention times, which increases the effective $L-d$ ratio and, consequently, the shape factor increases. ${ }^{1}$ The shape factors of the fractions outside of this length range were notably increased. With reference to the MALS scattering intensity and the UV absorbance signal, note that these particular fractions only represented $<4$ wt $\%$ of each particle collective. Complementary to the decrease of the shape factor with increasing particle length ranging between 130 and $270 \mathrm{~nm}$, a minor shift toward lower shape factors was observed with increasing ultrasound energy input. The notable decrease of the shape factor for $\mathrm{CNC}-40$ at rod lengths $>270 \mathrm{~nm}$ only constituted $1 \mathrm{wt} \%$ of the particle collective.

The overall shift of the CNC lengths with increasing ultrasound energy density, toward smaller values, and the concurrent narrowing of the PSD is shown in the cumulative mass-weighted length distribution plot in Fig. 8a. Equally, the rod length at $I_{\max }$ decreased, which is shown in Fig. $8 \mathrm{~b}$ for arbitrary particle diameters along with the size-related definition of CNCs, according to ISO/TS 20477 (ISO 2017b). ${ }^{2}$ The

\footnotetext{
${ }^{1} r_{g} r_{h}^{1}$ of rod-like nanostructures is $\sim 2$, while hard and soft spheres have shape factors of 0.778 and 0.977 , respectively (Brewer and Striegel 2009).

${ }^{2} \mathrm{CNCs}$ are defined as individual nano-objects with a diameter of 3-50 nm and a $L-d$ ratio of 5-50.
} 

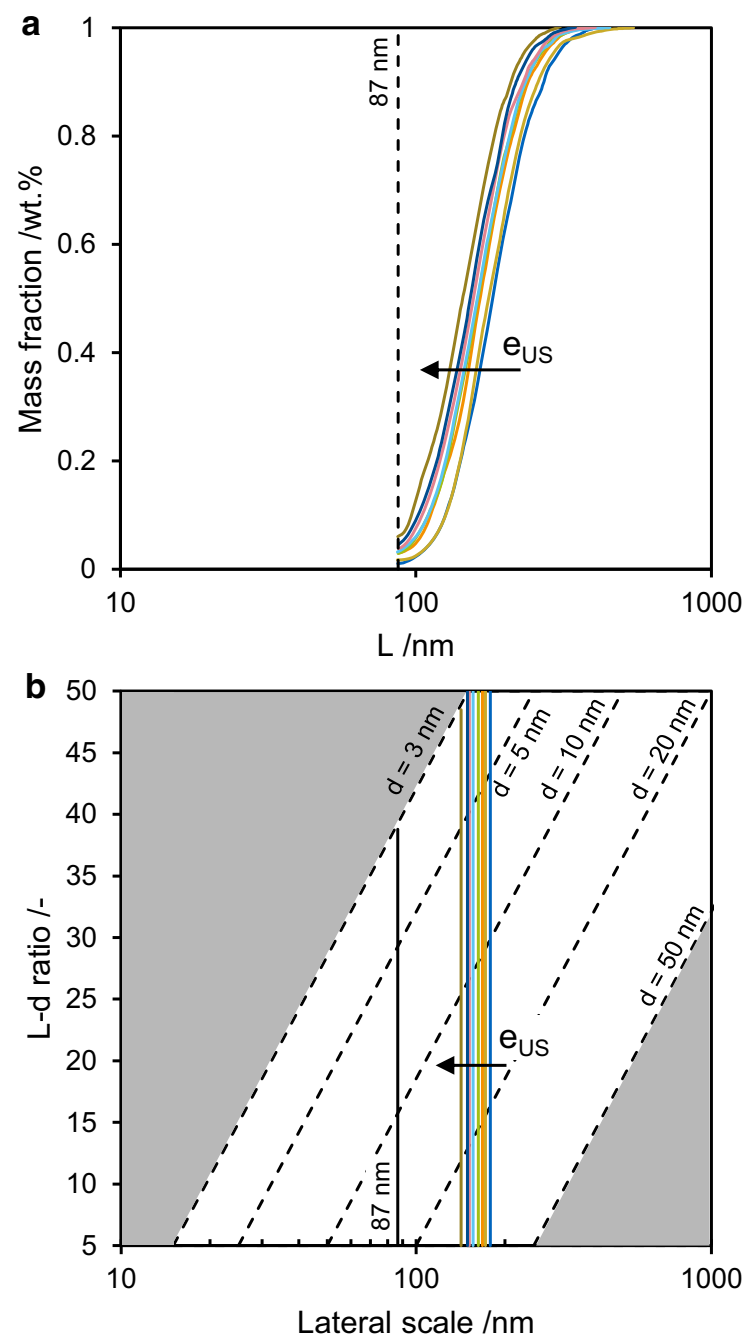

Fig. 8 a Cumulative mass-weighted length distribution of CNCs at different ultrasonication energy densities and $\mathbf{b}$ rod lengths at $I_{\max }$. The diagrams comprise the confinements of CNCs, according to ISO/TS 20477 (2017b), which describes them as nano-objects with diameters of 3-50 nm (dashed lines) and aspect ratios of 5-50 (vertical axis)

resolution limit of our set-up ( $87 \mathrm{~nm})$ is indicated by the vertical solid line in the figure. Provided that singles are infrangible, the dispersion of dimers can occur laterally, axially, or in a mixed form, depending on the arrangement of the nanostructure (Fig. 2). When laterally agglomerated dimers are dispersed the length is merely altered, whereas the diameter remains consistent when axially agglomerated dimers are dispersed. Figuratively translated to Fig. 8b, dispersion of laterally agglomerated dimers causes a vertical upward shift—-the diameter decreases and the $L$ - $d$ ratio increases. In contrast, dispersion of axially agglomerated dimers induces a diagonal downshift parallel to the dashed lines, which represent the particle diameter-length and $L-d$ ratio decrease. Similar considerations are applicable to the dispersion of clusters that have intrinsically higher $L-d$ ratios than dimers (Fig. 7).

In summary, extensive ultrasonication of $\mathrm{CNC}$ suspensions caused a progressive shift of the length distribution to shorter particle lengths and a concurrent narrowing of the distribution. Shorter CNCs had higher shape factors and, hence, lower diameters at similar length. Ultrasonication therefore facilitated $\mathrm{CNC}$ individualization by dispersing laterally and potentially present axially agglomerated nanostructures. Note that laterally agglomerated CNCs may not be perfectly congruent and, hence, a single deagglomeration event can cause a decrease of both particle length and particle diameter at the same time. Analogously, Beck et al. (2012), Beuguel et al. (2018), and Shojaeiarani et al. (2020) found that ultrasound treatment of $\mathrm{CNC}$ suspensions induces concurrent decrease of mean particle size and narrowing of particle size distribution, which then involves a reduction of the $\mathrm{L}-\mathrm{d}$ ratio.

Surface charge and colloidal stability of ultrasonicated CNCs

\section{Conductivity of CNC suspensions and surface charge density of $\mathrm{CNCs}$}

The electrical conductivity of the CNC stock suspension was measured in parallel to its incremental ultrasonication. The sample CNC-2 had a conductivity of $190.2 \pm 0.2 \mu \mathrm{S} \mathrm{cm}^{-1}$. The conductivity increased, following a power law, to $254.8 \pm 0.9 \mu \mathrm{S} \mathrm{cm}^{-1}$ (34\%) for CNC-40 (Fig. 9a). The background conductivity of water was $0.07 \pm 0.01 \mu \mathrm{S} \mathrm{cm}^{-1}$. An increase of $\sigma$ with increasing ultrasound energy density may have been attributed to the elevated diffusion coefficient of smaller particles and, in accordance with Fig. 1, the contribution of previously obscured surface charges in clusters, charged moieties that were released from the solvation shells of the particles, and potentially detached sulfate half-esters due to de-sulfation. For further insight into the origin of the increased electrical conductivity, each aliquot was treated with ionexchange resins to scavenge free ions from the 

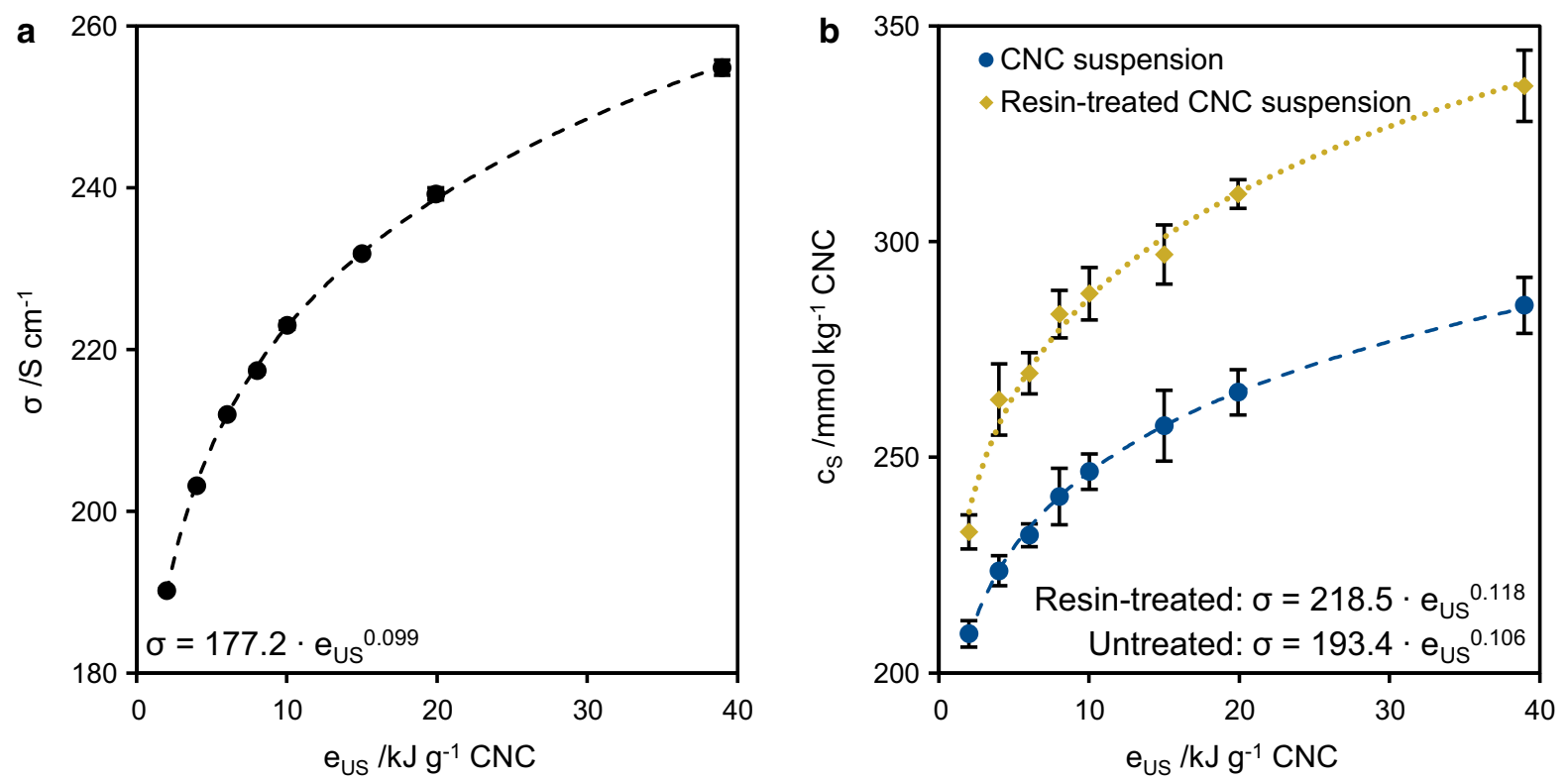

Fig. 9 a Electrical conductivity of $1 \mathrm{wt} \% \mathrm{CNC}$ at different stages of ultrasound treatment and b the corresponding surface sulfate charge of colloidal CNCs, along with ion-exchange resin-treated $\mathrm{CNC}$ suspensions at equal stages of ultrasonication

suspensions. Subsequently, the sulfate half-ester density of all samples was determined by conductometric titration (Fig. 9b). With increasing ultrasound energy density, an increase of the apparent sulfate half-ester density with power-law behavior, from $209 \pm 3 \mathrm{mmol} \mathrm{kg}^{-1} \quad \mathrm{CNC}$ for $\mathrm{CNC}-2$ to $285 \pm 7 \mathrm{mmol} \mathrm{kg}{ }^{-1} \mathrm{CNC}$ for CNC-40 (36\%), was observed. In the same interval, $c_{S}$ increased from $233 \pm 4 \mathrm{mmol} \mathrm{kg}^{-1} \mathrm{CNC}$ to $336 \pm 8 \mathrm{mmol} \mathrm{kg}^{-1}$ CNC (44\%) for ion-exchange resin-treated CNC suspensions. The mean apparent sulfate half-ester density of resin-treated suspensions was elevated overall by $16 \pm 2 \%$ compared to untreated suspensions. All measured values were in the range of commonly reported surface group densities for sulfated CNCs from cotton $\alpha$-cellulose, ranging from 80 to $350 \mathrm{mmol} \mathrm{kg}{ }^{-1} \mathrm{CNC}$ (Abitbol et al. 2013; Cherhal et al. 2015; Reid et al. 2017).

Each resin-treated CNC suspension was then separated with AF4 and analyzed by MALS and UV under equal measurement conditions as those for nontreated suspensions discussed earlier. No loss of sample mass was observed and congruent particle length distributions of non-treated and resin-treated CNC suspensions at each stage of ultrasonication implied that no adsorption of $\mathrm{CNCs}$ to the ionexchange resin occurred (Supporting Information,
Fig. S4). Furthermore, the hydrodynamic particle radius remained constant and, therefore, the shape factors were unaltered. In accordance with Abitbol et al. (2013) and Beck et al. (2015), it was concluded that the treatment of suspensions with ion-exchange resins promoted protonation of CNCs. Concurrently, constant hydrodynamic CNC properties suggested that no release of ionic species from the solvation shell or the particle surface occurred. Thus, the increase of both the electrical conductivity of the suspension and the increase of the apparent sulfate half-ester density with increasing ultrasound energy density was attributable to faster diffusion of smaller particles and exposure of surface charges, which were previously obscured in clusters (Girard et al. 2021).

\section{Colloidal stability}

Anionic surface sulfate groups on CNCs provide colloidal stability in aqueous suspensions by electrostatic particle repulsion. Their concentration is an important parameter for the manufacture of advanced materials with predictable and homogeneous properties. However, the concentration of surface sulfate half-esters provides no insight into colloidal stability and, instead, zeta potential measurements are employed (Foster et al. 2018). Herein, neither 
ultrasonication nor treatment with ion-exchange resins had significant effect on colloidal stability in terms of the zeta potential. The zeta potentials of CNCs treated with 2 and $40 \mathrm{~kJ} \mathrm{~g}^{-1} \mathrm{CNC}$, determined by ELS for the full particle collective and by EAF4 at $I_{\max }$, are shown in Table 2. All values were in the common range of -50 to $-20 \mathrm{mV}$ of sulfated CNCs (Foster et al. 2018). The elevated zeta potential of CNC-40-R was in the same stability regime as the other samples and all values indicated moderate colloidal stability $(\mathrm{Ku}-$ mar and Dixit 2017).

Stability of ultrasonicated CNC suspensions

All samples were hermetically sealed in glass vials and then conditioned at $4{ }^{\circ} \mathrm{C}$ under exclusion of light for 6 months. AF4-MALS-UV was subsequently applied to determine the long-term stability of ultrasonicated CNC suspensions. A comparison of the cumulative mass-weighted length distributions of colloidal CNCs, before and after conditioning, is shown in Fig. 10 for different extents of ultrasonication. Overall, no significant change of particle length distribution was observed. The mass-weighted particle fractions with lengths $<87 \mathrm{~nm}$ changed by $\pm 1 \mathrm{wt} \%$ (Supporting Information, Fig. S5) after 6 months and $L$ at $I_{\max }$ shifted to larger values by less than $2 \%$ for all samples. Concurrently, marginal broadening of the PSD, in terms of the $F W H M$, was noticeable. Figure 11 shows the related shape factors before and after conditioning. Generally, all samples exhibited lower shape factors after 6 months while the decline of $r_{g} r_{h}^{-1}$ was mitigated with increasing ultrasound energy density. In accordance with literature, CNCs are inferred to be susceptible to lateral agglomeration (Chen et al. 2020); however, extensive ultrasonication provided improved long-term stability.

\section{Conclusions and outlook}

This work presents, in detail, the effect of ultrasonication on the size distribution of colloidal CNCs from cotton $\alpha$-cellulose in aqueous suspensions. We applied AF4 with on-line coupled MALS and UV to analyze the particle dimensions in their native colloidal state. Furthermore, conductometric titration, EAF4, and ELS were applied to assess the surface charge density and the colloidal stability of ultrasonicated CNCs.

The results confirm that ultrasonication causes a decrease of mean particle length, along with a narrowing of particle length distribution. Increasing shape factors at shorter particle lengths indicate ultrasound-induced dispersion of dimers and clusters. The process conditions applied during ultrasound treatment did not cause liberation of ionic species from solvation shells of the particles and no de-sulfation was detected. Nevertheless, ultrasonication elevated the apparent sulfate half-ester density, which was attributed to a higher surface area by dispersion of clusters. Concurrently, colloidal stability remained constant throughout the experimental series. The stability of ultrasonicated CNC suspensions was assessed after conditioning the particles for 6 months in a benign environment. The impact of ultrasonication on PSD was found to be more lasting for high ultrasound energy densities, whereas CNCs ultrasonicated at low energy inputs form lateral agglomerates over the course of 6 months.

Ongoing endeavors directed at the commercialization of CNCs require comprehension and standardization of unit operations during $\mathrm{CNC}$ processing, as well as the implementation of consistent, reliable, and accurate characterization methods. In this context, our work addressed targeted ultrasonication of CNC suspensions to facilitate production of colloidally stable CNCs with narrow PSD and, thus, predictive and uniform properties. In addition, multi-detector

Table 2 Zeta potential of colloidal CNCs ultrasonicated at 2 and $40 \mathrm{~kJ} \mathrm{~g}^{-1} \mathrm{CNC}$

\begin{tabular}{llll}
\hline & $2 \mathrm{~kJ} \mathrm{~g}^{-1} \mathrm{CNC}$ & $40 \mathrm{~kJ} \mathrm{~g}^{-1} \mathrm{CNC}$ & \\
\cline { 2 - 4 } & $\zeta$ from ELS (off-line) $(\mathrm{mV})$ & $\zeta$ at $I_{\max }$ from EAF4 (on-line) $(\mathrm{mV})$ & $-27.5 \pm 5.1$ \\
\hline Untreated & $-31.4 \pm 1.8$ & $-29.1 \pm 4.9$ & $-36.8 \pm 2.8$ \\
Resin-treated & $-35.8 \pm 2.7$ & $-27.1 \pm 4.9$ & - \\
\hline
\end{tabular}




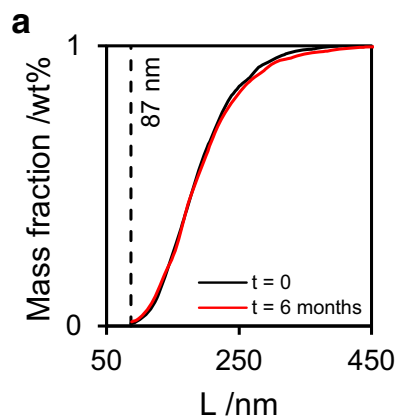

b

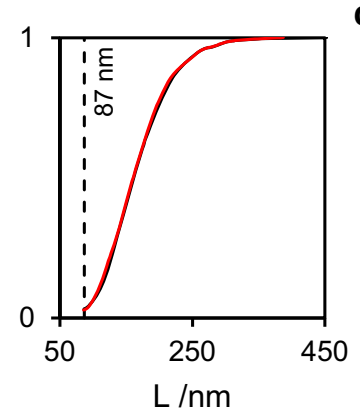

C

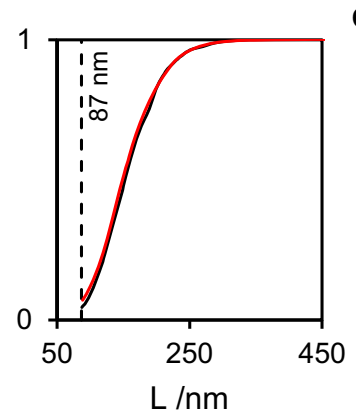

d

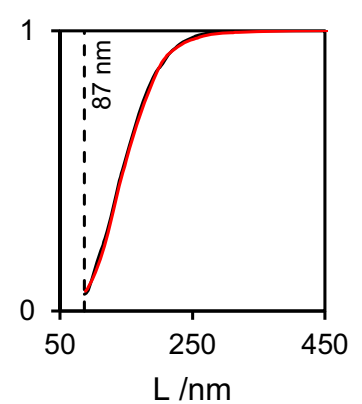

Fig. 10 Comparison of the cumulative mass-weighted length distributions of colloidal CNCs ultrasonicated at energy densities of a $2 \mathrm{~kJ} \mathrm{~g}^{-1} \mathrm{CNC}$, b $10 \mathrm{~kJ} \mathrm{~g}^{-1} \mathrm{CNC}, \mathbf{c} 20 \mathrm{~kJ} \mathrm{~g}^{-1} \mathrm{CNC}$, and d $40 \mathrm{~kJ} \mathrm{~g}^{-1} \mathrm{CNC}$ before and after storage for 6 months
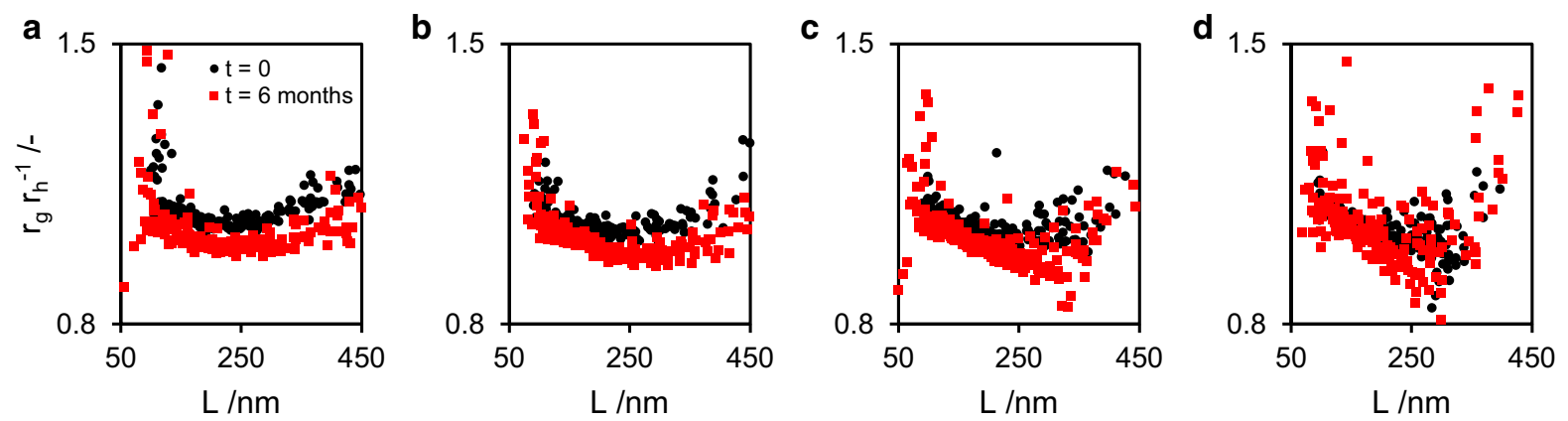

Fig. 11 Comparison of the shape factors of colloidal CNCs ultrasonicated at energy densities of a $2 \mathrm{~kJ} \mathrm{~g}^{-1} \mathrm{CNC}, \mathbf{b ~} 10 \mathrm{~kJ} \mathrm{~g}^{-1} \mathrm{CNC}$, c $20 \mathrm{~kJ} \mathrm{~g} \mathrm{~g}^{-1} \mathrm{CNC}$, and $\mathbf{d} 40 \mathrm{~kJ} \mathrm{~g}^{-1} \mathrm{CNC}$ before and after storage for 6 months

AF4 enables qualitative and quantitative characterization of CNCs in their native state.

Future work on ultrasonication of $\mathrm{CNC}$ suspension should address the combined effect of further process conditions - such as particle concentration, frequency, and amplitude - on size, colloidal stability, and the crystallinity index of CNCs. While our work suggests that no morphological degradation of CNCs occurs, Shojaeiarani et al. (2020) reported a notable decrease of crystallinity index by extensive ultrasonication. Therefore, investigating the structural impact of ultrasound treatment on CNCs is necessary to facilitate its targeted adaption to their required performance in advanced materials. Issues raised by our work that require further investigation involve sizerelated characterization of particle fractions with radii of gyration $r_{g}<25 \mathrm{~nm}$ via imaging techniques and the evaluation of agglomeration kinetics of protonated CNCs at different extents of ultrasonication.

\section{Supporting Information}

Validation of AF4 set-up, evolution of maximal MALS intensity, effect of CNC treatment with ionexchange resins, and fractograms after $\mathrm{CNC}$ conditioning for 6 months.

Acknowledgments The authors thank Johannes Zuber for his experimental contributions with CNC production and Alexandra Zandt and Jan Stotz for their assistance with conductometric titrations.

Author contributions CM and RD conceptualized and designed the study. Material preparation, data collection, and analysis were performed by CM and RD. FM and HB supervised the research. All authors contributed to the discussion and interpretation of results. The first draft of the manuscript was written by $\mathrm{CM}$, and all authors reviewed and edited successive versions. All authors read and approved the final manuscript.

Funding Open Access funding enabled and organized by Projekt DEAL. This research has been funded by the Federal Ministry of Education and Research of Germany (BMBF) in the framework of the NanoCELL project within the funding 
initiative NanoCare 4.0 (Grant Numbers 03XP196A and 03XP196B).

Availability of data and material All data are available from the authors upon reasonable request.

\section{Declarations}

Conflict of interest There are no conflicts of interest do declare.

Open Access This article is licensed under a Creative Commons Attribution 4.0 International License, which permits use, sharing, adaptation, distribution and reproduction in any medium or format, as long as you give appropriate credit to the original author(s) and the source, provide a link to the Creative Commons licence, and indicate if changes were made. The images or other third party material in this article are included in the article's Creative Commons licence, unless indicated otherwise in a credit line to the material. If material is not included in the article's Creative Commons licence and your intended use is not permitted by statutory regulation or exceeds the permitted use, you will need to obtain permission directly from the copyright holder. To view a copy of this licence, visit http://creativecommons.org/licenses/by/4.0/.

\section{References}

Abitbol T, Kloser E, Gray DG (2013) Estimation of the surface sulfur content of cellulose nanocrystals prepared by sulfuric acid hydrolysis. Cellulose 20(2):785-794. https://doi. org/10.1007/s10570-013-9871-0

Abitbol T, Rivkin A, Cao Y, Nevo Y, Abraham E, Ben-Shalom T, Lapidot S, Shoseyov O (2016) Nanocellulose, a tiny fiber with huge applications. Curr Opin Biotechnol 39:76-88. https://doi.org/10.1016/j.copbio.2016.01.002

Araki J, Wada M, Kuga S (2001) Steric stabilization of a cellulose microcrystal suspension by poly(ethylene glycol) grafting. Langmuir 17(1):21-27. https://doi.org/10.1021/ la001070m

Beck S, Bouchard J (2014) Auto-catalyzed acidic desulfation of cellulose nanocrystals. Nord Pulp Pap Res J 29(1):6-14. https://doi.org/10.3183/npprj-2014-29-01-p006-014

Beck S, Bouchard J, Berry R (2011) Controlling the reflection wavelength of iridescent solid films of nanocrystalline cellulose. Biomacromol 12(1):167-172. https://doi.org/10. 1021/bm1010905

Beck S, Bouchard J, Berry R (2012) Dispersibility in water of dried nanocrystalline cellulose. Biomacromol 13(5):1486-1494. https://doi.org/10.1021/bm300191k

Beck S, Méthot M, Bouchard J (2015) General procedure for determining cellulose nanocrystal sulfate half-ester content by conductometric titration. Cellulose 22(1):101-116. https://doi.org/10.1007/s10570-014-0513-y

Beck-Candanedo S, Roman M, Gray DG (2005) Effect of reaction conditions on the properties and behavior of wood cellulose nanocrystal suspensions. Biomacromol 6(2):1048-1054. https://doi.org/10.1021/bm049300p

Beuguel Q, Tavares JR, Carreau PJ, Heuzey M-C (2018) Ultrasonication of spray- and freeze-dried cellulose nanocrystals in water. J Colloid Interface Sci 516:23-33. https://doi.org/10.1016/j.jcis.2018.01.035

Boluk Y, Danumah C (2014) Analysis of cellulose nanocrystal rod lengths by dynamic light scattering and electron microscopy. J Nanoparticle Res. https://doi.org/10.1007/ s11051-013-2174-4

Bouchard J, Méthot M, Fraschini C, Beck S (2016) Effect of oligosaccharide deposition on the surface of cellulose nanocrystals as a function of acid hydrolysis temperature. Cellulose 23(6):3555-3567. https://doi.org/10.1007/ s10570-016-1036-5

Brewer AK, Striegel AM (2009) Particle size characterization by quadruple-detector hydrodynamic chromatography. Anal Bioanal Chem 393(1):295-302. https://doi.org/10. 1007/s00216-008-2319-y

Brinchi L, Cotana F, Fortunati E, Kenny JM (2013) Production of nanocrystalline cellulose from lignocellulosic biomass: technology and applications. Carbohydr Polym 94(1):154-169. https://doi.org/10.1016/j.carbpol.2013.01. 033

Brinkmann A, Chen M, Couillard M, Jakubek ZJ, Leng T, Johnston LJ (2016) Correlating cellulose nanocrystal particle size and surface area. Langmuir 32(24):6105-6114. https://doi.org/10.1021/acs.langmuir.6b01376

Campano C, Lopez-Exposito P, Gonzalez-Aguilera L, Blanco Á, Negro C (2021) In-depth characterization of the aggregation state of cellulose nanocrystals through analysis of transmission electron microscopy images. Carbohydr Polym 254:117271. https://doi.org/10.1016/j.carbpol. 2020.117271

Chen M, Parot J, Mukherjee A, Couillard M, Zou S, Hackley VA, Johnston LJ (2020) Characterization of size and aggregation for cellulose nanocrystal dispersions separated by asymmetrical-flow field-flow fractionation. Cellulose 27(4):2015-2028. https://doi.org/10.1007/s10570-01902909-9

Cherhal F, Cousin F, Capron I (2015) Influence of charge density and ionic strength on the aggregation process of cellulose nanocrystals in aqueous suspension, as revealed by small-angle neutron scattering. Langmuir 31(20):5596-5602. https://doi.org/10.1021/acs.langmuir. $5 \mathrm{~b} 00851$

Contado C (2017) Field flow fractionation techniques to explore the "nano-world." Anal Bioanal Chem 409(10):2501-2518. https://doi.org/10.1007/s00216-0170180-6

Cranston ED, Gray DG (2006) Morphological and optical characterization of polyelectrolyte multilayers incorporating nanocrystalline cellulose. Biomacromol 7(9):2522-2530. https://doi.org/10.1021/bm0602886

de Souza Lima MM, Wong JT, Paillet M, Borsali R, Pecora R (2003) Translational and rotational dynamics of rodlike cellulose whiskers. Langmuir 19(1):24-29. https://doi.org/ $10.1021 / 1 a 020475 z$

Dong XM, Gray DG (1997) Effect of counterions on ordered phase formation in suspensions of charged rodlike 
cellulose crystallites. Langmuir 13(8):2404-2409. https:// doi.org/10.1021/LA960724H

Dong XM, Revol J-F, Gray DG (1998) Effect of microcrystallite preparation conditions on the formation of colloid crystals of cellulose. Cellulose 5(1):19-32. https://doi.org/10.1023/ A:1009260511939

Drexel R, Siupa A, Carnell-Morris P, Carboni M, Sullivan J, Meier F (2020a) Fast and purification-free characterization of bio-nanoparticles in biological media by electrical asymmetrical flow field-flow fractionation hyphenated with multi-angle light scattering and nanoparticle tracking analysis detection. Molecules. https://doi.org/10.3390/ molecules 25204703

Drexel R, Sogne V, Dinkel M, Meier F, Klein T (2020b) Asymmetrical flow field-flow fractionation for sizing of gold nanoparticles in suspension. J vis Exp. https://doi.org/ $10.3791 / 61757$

Espinosa E, Sánchez R, Otero R, Domínguez-Robles J, Rodríguez A (2017) A comparative study of the suitability of different cereal straws for lignocellulose nanofibers isolation. Int J Biol Macromol 103:990-999. https://doi.org/10. 1016/j.ijbiomac.2017.05.156

Foster EJ, Moon RJ, Agarwal UP, Bortner MJ, Bras J, Camarero-Espinosa S, Chan KJ, Clift MJD, Cranston ED, Eichhorn SJ, Fox DM, Hamad WY, Heux L, Jean B, Korey M, Nieh W, Ong KJ, Reid MS, Renneckar S, Roberts R, Shatkin JA, Simonsen J, Stinson-Bagby K, Wanasekara N, Youngblood J (2018) Current characterization methods for cellulose nanomaterials. Chem Soc Rev 47(8):2609-2679. https://doi.org/10.1039/c6cs00895j

Fraschini C, Chauve G, Le Berre J-F, Ellis S, Méthot M, O'Connor B, Bouchard J (2014) Critical discussion of light scattering and microscopy techniques for CNC particle sizing. Nord Pulp Pap Res J 29(1):31-40. https://doi.org/ 10.3183/npprj-2014-29-01-p031-040

Gicquel E, Bras J, Rey C, Putaux J-L, Pignon F, Jean B, Martin C (2019) Impact of sonication on the rheological and colloidal properties of highly concentrated cellulose nanocrystal suspensions. Cellulose 26(13-14):7619-7634. https://doi.org/10.1007/s10570-019-02622-7

Girard M, Vidal D, Bertrand F, Tavares JR, Heuzey M-C (2021) Evidence-based guidelines for the ultrasonic dispersion of cellulose nanocrystals. Ultrason Sonochem 71:105378. https://doi.org/10.1016/j.ultsonch.2020.105378

Guan X, Cueto R, Russo P, Qi Y, Wu Q (2012) Asymmetric flow field-flow fractionation with multiangle light scattering detection for characterization of cellulose nanocrystals. Biomacromol 13(9):2671-2679

Habibi Y, Lucia LA, Rojas OJ (2010) Cellulose nanocrystals: chemistry, self-assembly, and applications. Chem Rev 110(6):3479-3500. https://doi.org/10.1021/cr900339w

Haouache S, Karam A, Chave T, Clarhaut J, Amaniampong PN, Garcia Fernandez JM, de Oliveira Vigier K, Capron I, Jérôme F (2020) Selective radical depolymerization of cellulose to glucose induced by high frequency ultrasound. Chem Sci 11(10):2664-2669. https://doi.org/10.1039/ DOSC00020E

ISO (2017a) ISO 22412:2017: particle size analysis—dynamic light scattering (DLS), 2017-02. International Organization for Standardization, Geneva, Switzerland
ISO (2017b) ISO/TS 20477:2017: nanotechnologies—standard terms and their definition for cellulose nanomaterial. 2017-10. International Organization for Standardization, Geneva, Switzerland

ISO (2018a) ISO/CD TS 23151: nanotechnologies-particle size distribution for cellulose nanocrystals (under development). International Organization for Standardization, Geneva, Switzerland

ISO (2018b) ISO/TS 21362:2018: nanotechnologies—analysis of nanoobjects using asymmetrical-flow and centrifugal field-flow fractionation. International Organization for Standardization, Geneva, Switzerland

Jakubek ZJ, Chen M, Couillard M, Leng T, Liu L, Zou S, Baxa U, Clogston JD, Hamad WY, Johnston LJ (2018) Characterization challenges for a cellulose nanocrystal reference material: dispersion and particle size distributions. J Nanoparticle Res. https://doi.org/10.1007/s11051-0184194-6

Jiang F, Esker AR, Roman M (2010) Acid-catalyzed and sol-

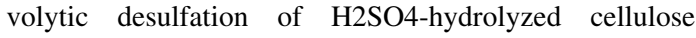
nanocrystals. Langmuir 26(23):17919-17925. https://doi. org/10.1021/la1028405

Jonoobi M, Oladi R, Davoudpour Y, Oksman K, Dufresne A, Hamzeh Y, Davoodi R (2015) Different preparation methods and properties of nanostructured cellulose from various natural resources and residues: a review. Cellulose 22(2):935-969. https://doi.org/10.1007/s10570-015-05510

Kaushik M, Chen WC, van Ven TGM, de Moores A (2014) An improved methodology for imaging cellulose nanocrystals by transmission electron microscopy. Nord Pulp Pap Res J 29(1):77-84. https://doi.org/10.3183/npprj-2014-29-01p077-084

Kontturi E (2018) Supramolecular aspects of native cellulose: fringed-fibrillar model, leveling-off degree of polymerization and production of cellulose nanocrystals. In: Potthast A, Hell J, Rosenau T (eds) Cellulose science and technology: chemistry, analysis, and applications. Wiley, Hoboken, pp 263-276

Kumar A, Dixit CK (2017) Methods for characterization of nanoparticles. In: Nimesh S, Chandra R, Gupta N (eds) Advances in nanomedicine for the delivery of therapeutic nucleic acids. Elsevier Science, Kent, pp 43-58

Lin K-H, Hu D, Sugimoto T, Chang F-C, Kobayashi M, Enomae $\mathrm{T}$ (2019) An analysis on the electrophoretic mobility of cellulose nanocrystals as thin cylinders: relaxation and end effect. RSC Adv 9(58):34032-34038. https://doi.org/10. 1039/c9ra05156b

Litzen A, Wahlund KG (1991) Zone broadening and dilution in rectangular and trapezoidal asymmetrical flow field-flow fractionation channels. Anal Chem 63(10):1001-1007. https://doi.org/10.1021/ac00010a013

Marchessault R, Morehead F, Koch M (1961) Some hydrodynamic properties of neutral suspensions of cellulose crystallites as related to size and shape. J Colloid Sci 16(4):327-344. 8522(61)90033-2

Mazloumi M, Johnston LJ, Jakubek ZJ (2018) Dispersion, stability and size measurements for cellulose nanocrystals by static multiple light scattering. Cellulose 
25(10):5751-5768. https://doi.org/10.1007/s10570-0181961-6

Metzger C, Auber D, Dähnhardt-Pfeiffer S, Briesen H (2020) Agglomeration of cellulose nanocrystals: the effect of secondary sulfates and their use in product separation. Cellulose. https://doi.org/10.1007/s10570-020-03476-0

Moon RJ, Martini A, Nairn J, Simonsen J, Youngblood J (2011) Cellulose nanomaterials review: structure, properties and nanocomposites. Chem Soc Rev 40(7):3941-3994. https:// doi.org/10.1039/c0cs00108b

Mukherjee A, Hackley VA (2018) Separation and characterization of cellulose nanocrystals by multi-detector asymmetrical-flow field-flow fractionation. Analyst 143(3):731-740. https://doi.org/10.1039/c7an01739a

Nickerson RF, Habrle JA (1947) Cellulose intercrystalline structure. Ind Eng Chem 39(11):1507-1512. https://doi. org/10.1021/ie50455a024

NIST (2021) SRM 1964: Polystyrene Spheres (Nominal Diameter $60 \mathrm{~nm}$ )

Phan-Xuan T, Thuresson A, Skepö M, Labrador A, Bordes R, Matic A (2016) Aggregation behavior of aqueous cellulose nanocrystals: the effect of inorganic salts. Cellulose 23(6):3653-3663. https://doi.org/10.1007/s10570-0161080-1

Postnova Analytics GmbH (2020a) NovaAnalysis (2007). https://www.postnova.com/

Postnova Analytics GmbH (2020b) NovaFFF (2.1.0.5). https:// www.postnova.com/

Postnova Analytics GmbH (2020c) NovaMALS (1.5.0.8). https://www.postnova.com/

Rånby BG, Ribi E (1950) Ueber den Feinbau der Zellulose (Ultrastructure of cellulose). Experientia 6(1):12-14. https://doi.org/10.1007/BF02154044

Reid MS, Villalobos M, Cranston ED (2017) Benchmarking cellulose nanocrystals: from the laboratory to industrial production. Langmuir 33(7):1583-1598. https://doi.org/ 10.1021/acs.langmuir.6b03765

Revol J-F, Godbout L, Dong X-M, Gray DG, Chanzy H, Maret G (1994) Chiral nematic suspensions of cellulose crystallites; phase separation and magnetic field orientation. Liq Cryst 16(1):127-134. https://doi.org/10.1080/ 02678299408036525

Roman M (2015) Toxicity of Cellulose Nanocrystals: A Review. Ind Biotechnol 11(1):25-33. https://doi.org/10. 1089/ind.2014.0024

Rudie A (2017) Commercialization of cellulose nanofibril (CNF) and cellulose nanocrystal (CNC): pathway and challenges. In: Kargarzadeh H, Ahmad I, Thomas S, Dufresne A (eds) Handbook of nanocellulose and cellulose nanocomposites, vol 1. Wiley-VCH. Weinheim, Germany, pp 761-797

Ruiz-Palomero C, Laura Soriano M, Valcárcel M (2017) Detection of nanocellulose in commercial products and its size characterization using asymmetric flow field-flow fractionation. Microchim Acta 184(4):1069-1076. https:// doi.org/10.1007/s00604-017-2106-6

Shafiei-Sabet S, Hamad WY, Hatzikiriakos SG (2012) Rheology of nanocrystalline cellulose aqueous suspensions. Langmuir 28(49):17124-17133. https://doi.org/10.1021/ la303380v
Shatkin JA, Kim B (2015) Cellulose nanomaterials: life cycle risk assessment, and environmental health and safety roadmap. Environ Sci Nano 2(5):477-499. https://doi.org/ 10.1039/C5EN00059A

Shojaeiarani J, Bajwa D, Holt G (2020) Sonication amplitude and processing time influence the cellulose nanocrystals morphology and dispersion. Nanocomposites 6(1):41-46. https://doi.org/10.1080/20550324.2019.1710974

Stepto R, Chang T, Kratochvíl P, Hess M, Horie K, Sato T, Vohlídal J (2015) Definitions of terms relating to individual macromolecules, macromolecular assemblies, polymer solutions, and amorphous bulk polymers (IUPAC Recommendations 2014). Pure Appl Chem 87(1):71-120. https://doi.org/10.1515/pac-2013-0201

Stepto RFT (2009) Dispersity in polymer science (IUPAC Recommendations 2009). Pure Appl Chem 81(2):351-353. https://doi.org/10.1351/PAC-REC-08-05-02

Trache D, Hussin MH, Haafiz MKM, Thakur VK (2017) Recent progress in cellulose nanocrystals: sources and production. Nanoscale 9(5):1763-1786. https://doi.org/10.1039/ c6nr09494e

van de Hulst HC (1958) Light scattering by small particles. Q J R Meteorol Soc 84(360):198-199. https://doi.org/10.1002/ qj.49708436025

Vanderfleet OM, Cranston ED (2020) Production routes to tailor the performance of cellulose nanocrystals. Nat Rev Mater. https://doi.org/10.1038/s41578-020-00239-y

von Smoluchowski M (1906) Zur kinetischen Theorie der Brownschen Molekularbewegung und der Suspensionen. Ann Phys 326(14):756-780. https://doi.org/10.1002/andp. 19063261405

Wahlund KG, Giddings JC (1987) Properties of an asymmetrical flow field-flow fractionation channel having one permeable wall. Anal Chem 59(9):1332-1339. https://doi.org/ 10.1021/ac00136a016

Wang N, Ding E, Cheng R (2007) Thermal degradation behaviors of spherical cellulose nanocrystals with sulfate groups. Polymer 48(12):3486-3493. https://doi.org/10. 1016/j.polymer.2007.03.062

Wang N, Ding E, Cheng R (2008) Preparation and liquid crystalline properties of spherical cellulose nanocrystals. Langmuir 24(1):5-8. https://doi.org/10.1021/la702923w

Wang QQ, Zhu JY, Reiner RS, Verrill SP, Baxa U, McNeil SE (2012) Approaching zero cellulose loss in cellulose nanocrystal $(\mathrm{CNC})$ production: recovery and characterization of cellulosic solid residues (CSR) and CNC. Cellulose 19(6):2033-2047. https://doi.org/10.1007/s10570012-9765-6

Zianor Azrina ZA, Beg MDH, Rosli MY, Ramli R, Junadi N, Alam AKMM (2017) Spherical nanocrystalline cellulose (NCC) from oil palm empty fruit bunch pulp via ultrasound assisted hydrolysis. Carbohydr Polym 162:115-120. https://doi.org/10.1016/j.carbpol.2017.01.035

Publisher's Note Springer Nature remains neutral with regard to jurisdictional claims in published maps and institutional affiliations. 